\title{
Effect of Lactobacillus casei on the production of pro-inflammatory markers in streptozotocin-induced diabetic rats.
}

\begin{abstract}
It has been demonstrated that probiotic supplementation has positive effects in several murine models of disease through influences on host immune responses. This study examined the effect of Lactobacillus casei strain Shirota (L. casei Shirota) on the blood glucose, C-reactive protein (CRP), Interleukin-6 (IL-6), Interleukin-4 (IL-4), and body weight among STZinduced diabetic rats. Diabetes mellitus was induced by streptozotocin (STZ, $50 \mathrm{mg} / \mathrm{kg} \mathrm{BW}$ ) in male Sprague-Dawley rats. Streptozotocin caused a significant increase in the blood glucose levels, CRP, and IL-6. L. casei Shirota supplementation lowered the CRP and IL-6 levels but had no significant effect on the blood glucose levels, body weight, or IL-4. Inflammation was determined histologically. The presence of the innate immune cells was not detectable in the liver of L. casei Shirota-treated hyperglycemic rats. The probiotic L. casei Shirota significantly lowered blood levels of pro-inflammatory cytokines (IL-6, CRP) and neutrophils in diabetic rats, showing a lower risk of diabetes mellitus and its complications.
\end{abstract}

Keyword: Diabetes mellitus; Inflammatory biomarkers; Probiotics; Lactobacillus casei Shirota. 